\title{
Effects of COVID-19 pandemic on general surgical emergencies: are some emergencies really urgent? Level 1 trauma center experience
}

\author{
Ahmet Surek $^{1}$ (D) Sina Ferahman ${ }^{1} \cdot$ Eyup Gemici $^{1} \cdot$ Ahmet Cem Dural $^{1} \cdot$ Turgut Donmez $^{1} \cdot$ Mehmet Karabulut $^{1}$
}

Received: 6 June 2020 / Accepted: 17 October 2020 / Published online: 2 November 2020

(c) Springer-Verlag GmbH Germany, part of Springer Nature 2020

\begin{abstract}
Purpose The aim of this paper is to investigate the effect of COVID-19 pandemic on general surgical emergencies as well as analyzing the effectiveness of measures taken in reducing the incidence of COVID-19 in patients and healthcare professionals. Methods Patients who underwent emergency surgery between the pandemic period of March 14th to May 15th 2020 and within the same period from the previous year were reviewed retrospectively. COVID-19 incidence in patients and health professionals working in the general surgery department during these periods was questioned.

Results Demographic data were similar between the two time periods. The number of patients who underwent surgery in the pandemic group $(n=103)$ was lower than the control group $(n=252)$. There was a 59.1\% reduction in emergency surgeries. The biggest decreases were the admissions of incarcerated hernia, uncomplicated appendicitis and acute cholecystitis (92\%, $81.3 \%, 47.3 \%$, respectively). During the pandemic, an increase was of patient rates who underwent surgery for complicated appendicitis and AMIO $(p=0.001, p=0.019$, respectively). The rate of mortality was higher in patients who underwent emergency surgery during pandemic $(p=0.049)$. The results of COVID-19 screening were positive in $6(6 / 103,5.82 \%)$ patients undergoing emergency surgery. None of the doctors working in the ward were infected with COVID-19 infection $(0 / 20)$. The screening tests were positive in only two nurses working on the ward $(2 / 24,8.33 \%)$.

Conclusion In this and similar pandemics, we suggest that a new algorithm is necessary to approach emergencies and the results of this study can contribute to that end.
\end{abstract}

Keywords COVID-19 $\cdot$ General surgery $\cdot$ Emergency $\cdot$ Operation

\section{Introduction}

Coronavirus disease 2019 (COVID-19) is an infectious respiratory disease caused by the new virus, severe acute respiratory syndrome coronavirus 2 (SARS-CoV-2). Since its appearance in December 2019, the COVID-19 infection has spread globally and has been declared a pandemic by the World Health Organization on March 11, 2020 [1, 2]. SARS-CoV-2 is characterized by respiratory symptoms such as fever, dry cough, shortness of breath and infiltration in chest X-ray [3]. Despite the mild or moderate prognosis in most COVID-19 patients, up to $5-10 \%$ can follow a

Ahmet Surek

ahmetsurek82@hotmail.com

1 Department of General Surgery, Turkish Ministry of Health, University of Health Sciences (UHS), Bakirkoy Dr. Sadi Konuk Training and Research Hospital, Building A, Level 4, Tevfik Saglam Cad. Nr:11, Bakirkoy, Istanbul, Turkey severe, potentially life-threatening course [4]. In addition to the risk of death, due to the rapid spread of the pandemic, easy transmission from person to person and high mortality rate, it brought unbearable psychological pressures and concerns [5]. In this context, there may have been a decrease in patients' application to hospitals even in emergencies. There is a reported $38 \%$ reduction in US cardiac catheterization laboratory ST-Elevation Myocardial Infarction (STEMI) which occurred in the early stage of the COVID-19 pandemic. Similar results were reported in Spain with a $40 \%$ reduction [6-8].

COVID-19 infection was detected for the first time in Turkey on March 11th and the first death took place on March 18th. The number of non-COVID-19 patients admitted to hospitals decreased after the number of cases started to increase and deaths related to the disease started to appear. However, the rapid spread of the infection caused a drastically increase for the need of beds in hospitals. During this period, European Association for Trauma and Emergency 
Surgery (ESTES) recommended postponing elective cases to a later date, except for some identified cases, and a series of recommendations for perioperative preparation for emergency surgery and trauma patients were reported [9]. These recommendations guided us through the challenges we followed during the early stage of the pandemic. Also, the data reveal that the hospital-acquired virus was recorded to be $41 \%$ among patients during the COVID-19 pandemic [10]. According to these recommendations, we have postponed our elective surgeries, except for cancer cases, and reduced the surgical admissions in outpatient clinics and wards to protect the patient and staff health and meet the increased need for bed. Emergency cases requiring surgery were admitted without any interruption during that period. However, a significant reduction occurred in general hospital admissions.

In this study, we compared the emergency surgeries performed in our general surgery clinic during the COVID-19 pandemic period with the same period of last year. We aimed to investigate the effect of COVID-19 outbreak on emergency surgeries. For a second progress report of the study, we analyzed the effectiveness of the measures we have taken and the incidence of COVID-19 of patients and healthcare professionals.

\section{Materials and methods}

In the pandemic period between March 14 and May 15, 2020, and in the same period of the previous year, the files of patients who underwent emergency surgery and followed up non-operatively were reviewed retrospectively. All surgical procedures performed on patients requiring surgery in the first $24 \mathrm{~h}$ of admission and those who need an urgent surgery during non-operative follow-up in the emergency department are categorized as "emergency surgery" in this study. Also, patients who followed non-operatively by surgeons in emergency department were included to study for the analysis of two study periods. Patients who were urgently re-operated due to complications after elective surgery were excluded from the study. Demographic data, number of surgeries, type of surgery, surgical findings, re-operations, morbidity and mortality rates were recorded. Patients who underwent emergency surgery in the pandemic period in 2020 were named as "Pandemic Group" and patients who underwent emergency operations during the same period in 2019 were named as "Control Group". The data obtained were compared between the two groups.

As a secondary outcome, patients were investigated for COVID-19 infection. Those who had pre-operative COVID-19 infection and those who had postoperative hospital-induced COVID-19 infection were investigated. Preoperative chest tomography was performed in all patients who underwent emergency surgery in the Pandemic Group. The patients with findings consistent in pre-operative chest tomography with COVID-19 pneumonia and patients with symptoms suggestive of COVID-19 infection were PCR tested and these patients were hospitalized in isolated rooms in surgery ward. In addition, chest tomography and PCR tests were performed on patients with postoperative clinical findings. Finally, the doctors, ward and operation room nurses, transport personnel and other healthcare professionals who had contact with these patients were screened for the COVID-19 infection.

\section{Statistical analysis}

Statistical analysis was performed with Statistical Package for the Social Sciences (SPSS) program version 24.0 (IBM Corp, Armonk, NY). Patient characteristics were analyzed via descriptive statistics. For continuous variables, the mean and standard derivation or median and interquartile ranges were calculated and Student's $t$ test was used for the analysis of two groups. For categorical variables, the numbers and percentages in each category were recorded and variables were compared with Pearson chi-square or Fisher's exact test for suitable parameters. $P$ values of 0.05 or below were accepted as statistically significant.

\section{Results}

Within the two periods in this study's scope, a total of 453 patients, including 355 patients who underwent emergency surgery and 98 patients who were followed up nonoperatively, were included in present study. There was a 59.1\% reduction in the number of emergency surgeries and 50\% decrease in the number of non-operatively followed patients in the Pandemic Group. The demographic data and the number of patients who underwent surgery for trauma, GIS bleeding, perforation of gastrointestinal tract and mesenteric ischemia in the two groups were similar (Table 1).

In the Pandemic Group, the rate of surgery was statistically higher in patients who were admitted to hospital for AMIO (Table 1). There was a severe decrease in the number of patients followed up non-operatively. When the etiological reasons were examined, the admissions for adhesive bowel obstruction were quite low in the Pandemic Group (15 to 2 ).

Although in the Pandemic Group, there were 92\% fewer surgeries performed for incarcerated hernia; there was no statistically significant difference in the patients who underwent small bowel resection between the two groups (Table 1). 
Table 1 Demographic data and comparison of patients who underwent emergency surgery and patients were who followed non-operatively

\begin{tabular}{|c|c|c|c|}
\hline & Control group & Pandemic group & $P$ value \\
\hline Age $($ mean \pm SD) & $46.1 \pm 17.9$ & $46.6 \pm 18.9$ & ${ }^{\mathrm{a}} 0.890$ \\
\hline Gender & & & b0.928 \\
\hline Male, $n(\%)$ & $160(63.5)$ & $67(65)$ & \\
\hline Female, $n(\%)$ & $92(36.5)$ & $36(35)$ & \\
\hline Trauma (Total), $n$ & 11 & 12 & ${ }^{c} 0.863$ \\
\hline Blunt trauma, $n(\%)$ & $3(27.3)$ & $3(25)$ & \\
\hline Gunshot wound, $n(\%)$ & $3(27.3)$ & $2(16.7)$ & \\
\hline Stab wound, $n(\%)$ & $5(45.5)$ & $7(58.3)$ & \\
\hline GIS bleeding (Total), $n$ & 9 & 7 & ${ }^{c} 0.902$ \\
\hline Surgery performed, $n(\%)$ & $3(33.3)$ & $2(35)$ & \\
\hline Nonoperatively followed, $n(\%)$ & $6(66.7)$ & $5(65)$ & \\
\hline Acute mesenteric ischemia (Total), $n$ & 2 & 2 & NS \\
\hline Perforations (Total), $n$ & 14 & 18 & ${ }^{\mathrm{c}} 0.892$ \\
\hline Peptic ulcus, $n(\%)$ & $6(42.9)$ & $9(50)$ & \\
\hline Small intestine, $n(\%)$ & $2(14.3)$ & $3(16.7)$ & \\
\hline Colon, $n(\%)$ & $6(42.9)$ & $6(33.3)$ & \\
\hline AMIO (Total), $n$ & 46 & 24 & ${ }^{\mathrm{c}} 0.019$ \\
\hline Surgery performed, $n(\%)$ & $25(54.3)$ & $20(83.3)$ & \\
\hline Nonoperatively followed, $n(\%)$ & $21(45.7)$ & $4(16.7)$ & \\
\hline Incarcerated hernia (Total), $n$ & 25 & 2 & ${ }^{c} 0.279$ \\
\hline Without resection, $n(\%)$ & $22(88)$ & $1(50)$ & \\
\hline With resection, $n(\%)$ & $3(12)$ & $1(50)$ & \\
\hline Acute cholecystitis (Total), $n$ & 55 & 29 & ${ }^{c} 0.203$ \\
\hline Surgery performed, $n(\%)$ & $17(30.9)$ & $5(17.2)$ & \\
\hline Nonoperatively followed, $n(\%)$ & $38(69.1)$ & $24(82.8)$ & \\
\hline Acute appendicitis (Total), $n$ & 155 & 42 & ${ }^{\mathrm{c}} 0.001$ \\
\hline Uncomplicated, $n(\%)$ & $126(83.2)$ & $24(57.1)$ & \\
\hline Complicated, $n(\%)$ & $29(16.8)$ & $18(42.9)$ & \\
\hline
\end{tabular}

GIS gastrointestinal system, $A M I O$ acute mechanical intestinal obstruction

${ }^{\text {a S}}$ Student's $t$ test

${ }^{\mathrm{b}}$ Pearson chi-square test

${ }^{c}$ Fisher's exact test
During the pandemic period, the number of patients admitted for acute cholecystitis decreased by $47.3 \%$. Although the rate of surgery was higher in the Control Group, it was statistically insignificant (Table 1).

In the Pandemic Group, there was a $73 \%$ reduction in the number of patients who underwent surgery for acute appendicitis. Admissions for uncomplicated appendicitis decreased by $81.3 \%$, and admissions for complicated appendicitis decreased by $38 \%$. However, the rate of patient who underwent surgery for complicated appendicitis was statistically higher in the Pandemic Group (Table 1).

While the total postoperative complications and reoperations were similar between the two groups, the rate of mortality was statistically higher in the Pandemic Group (Table 2).

As a secondary outcome, in preoperative evaluation, findings compatible with COVID-19 infection were observed in chest tomography of six $(5.8 \%)$ patients. PCR test of two (1.94\%) of these patients was determined positive. These patients were consulted with the infectious diseases clinic and received COVID-19 treatment. None of the patients deceased. In one patient without any initial COVID-19 signs and symptoms, findings consistent with COVID-19 infection were observed in chest tomography performed during postoperative intensive care follow-up. PCR test of this patient was negative. This patient was determined ASA 4 and was admitted to the postoperative intensive care unit. The patient deceased on the $5^{\text {th }}$ postoperative day. None of the doctors who working on the ward and none of the healthcare professional participating in emergency surgeries were infected with COVID-19 infection. However, COVID-19 infection was detected in two $(8.3 \%)$ nurses working in our ward. 
Table 2 Short-term surgical outcomes of patients who underwent emergency surgery

\begin{tabular}{lccc}
\hline & $\begin{array}{l}\text { Control group } \\
(n=252)\end{array}$ & $\begin{array}{l}\text { Pandemic group } \\
(n=103)\end{array}$ & $P$ value \\
\hline Total post-operative complications, $n(\%)$ & $43(17.06)$ & $25(24.27)$ & ${ }^{c} 0.137$ \\
Cardiovascular system & $4(1.58)$ & $2(1.94)$ & \\
Pulmonary system & $6(2.38)$ & $4(3.88)$ & \\
Urinary system & $2(0.79)$ & $1(0.97)$ & \\
Post-operative hemorrhage & $3(1.19)$ & $1(0.97)$ & \\
Prolonged ileus & $5(1.98)$ & $4(3.88)$ & \\
Superficial wound infection & $11(4.36)$ & $6(5.82)$ & \\
Wound dehiscence & $3(1.19)$ & $2(1.94)$ & \\
Intra-abdominal abscess & $6(2.38)$ & $3(2.91)$ & \\
Anastomotic leakage & $3(1.19)$ & $2(1.94)$ & \\
Re-operations, $n(\%)$ & $6(2.38)$ & $4(3.88)$ & ${ }^{c} 0.722$ \\
Mortality, $n(\%)$ & $3(1.19)$ & $5(4.85)$ & \\
\hline
\end{tabular}

${ }^{\mathrm{c}}$ Fisher's exact test

\section{Discussion}

The number of emergency surgeries decreased by $59.1 \%$ during the pandemic period. The biggest decreases observed were in incarcerated hernia, uncomplicated appendicitis and acute cholecystitis. The number of patients who underwent surgery for trauma, gastrointestinal system bleeding, acute mesenteric ischemia and gastrointestinal perforation were similar in both groups. During pandemic, an increase in the rate of surgeries for complicated appendicitis and AMIO was observed. In addition, the rate of mortality was higher in patients who underwent emergency surgery in the pandemic period.

ESTES recommended that the management for trauma and emergency general surgery operations should be the same as in the non-pandemic period [9]. Although we follow this recommendation, the number of emergency surgeries decreased significantly during the pandemic. A study by Gallego et al. [11] reported that during the pandemic, the number of general surgery emergency interventions decreased from 3.6 to 1.6 a day on average. In present study, the surgeries performed for uncomplicated appendicitis, acute cholecystitis and incarcerated hernia were affected the most by the pandemic. The decrease in the number of surgeries for uncomplicated appendicitis and acute cholecystitis may be due to the more antibiotic treatment administered during pandemic or some patients could have recovered with symptomatic treatment in quarantine. Although laparoscopic surgery is the gold standard treatment for uncomplicated appendicitis and acute cholecystitis, antibiotic therapy is an option $[12,13]$. In addition, a multi-center study by Tankel et al. [14] states that admissions of acute appendicitis decreased by $40 \%$ during the pandemic period, and concluded that patients with possibly mild appendicitis recovered with symptomatic treatment at home. Although there was a $92 \%$ reduction in the number of surgeries for incarcerated hernias, the proportion of patients requiring small bowel resection remained the same. Nevertheless, in present study, the emergency surgeries performed due to trauma, GIS bleeding, perforation and mesenteric ischemia were not affected by the pandemic. One of similar the features of these emergencies were that they are in the emergency surgery category that requires intervention within several hours or immediately which shows high mortality if early intervention is not performed [15]. Similarly, in a study by Madelona et al. [16], although there was a decrease in total urological emergencies, number of admissions requiring immediate intervention such as testicular torsion, acute obstructive pyelonephritis and acute obstructive renal injury did not decrease. These results reveal that patients who really need emergency surgery are applied to the hospital during pandemic.

The rate of surgeries performed for complicated appendicitis and AMIO increased statistically significantly during the pandemic period. Gallego et al. [11] reported that they performed only four appendectomy in a 20-day period during the pandemic and all cases were complicated appendicitis. Similarly, in a multicenter retrospective study, it was reported that the rate of surgery performed for AMIO increased during the pandemic [17]. In present study, one of the reasons for the increase in the rate of surgeries performed for AMIO was the $86 \%$ decrease in hospital admissions for adhesive intestinal obstruction, for which non-operative follow-up is successful. World Society of Emergency Surgery (WSES) recommended that these patients be followed up non-operatively in the absence of signs of peritonitis or bowel ischemia and success rates have been reported between 70 and $90 \%$ [18]. One of the important results of this study is that although the rates of reoperation and morbidity were similar in patients who underwent emergency 
surgery between the both periods, the rate of mortality was higher during pandemic. The study of Cano-Valderrama et al. [17] reported the rate of morbidity was higher in patients who underwent emergency surgery during the pandemic, while mortality and reoperation rates were similar. This may be due to the delayed admission of patients to hospitals during the pandemic period. In a multicenter study, delayed treatment was reported in more than $40 \%$ of nontraumatic abdominal emergencies during pandemic [19].

De Simone et al. [20] reported that non-operative approach can be applied acute appendicitis, acute cholecystitis, adhesive small bowel obstructions and incarcerated hernias in pandemic period. East et al. [21] reported that the use of taxis procedure (a manual reduction of hernia under analgesia/sedation) for incarcerated inguinal hernia is a useful first-line treatment in situations where surgical care is not immediately available, including the COVID-19 pandemic. In this study, there was a severe decrease in the number of these patients who were recommended nonoperative follow-up in recent publications during pandemic. Especially in these pandemic situations, we wonder whether outpatient follow-up is possible in these patient groups, where nonsurgical follow-ups are successful to meet the increasing bed need in hospitals.

As a secondary outcome, the effectiveness of the measures taken to protect against COVID-19 infection was investigated. Preoperative chest tomography was performed in all patients who underwent emergency surgery. Preoperative PCR test was not routinely performed due to delayed test results. ESTES stated that the delay in PCR test results may affect the time-critical operative management of surgical conditions [9]. In addition, studies have reported that chest tomography is more sensitive than PCR test $[22,23]$. The patients who had clinical suspicion and positive tomography findings were isolated and PCR tested. A special operating room and elevator are designed only for patients with suspected COVID-19. During operations of these patients, our surgical team absolutely used N95/FFP3 masks, protective overalls and eye visors. No doctors or members of the surgical team were infected with COVID-19 on account of the measures we took in line with the recommendations of ESTES [9]. In the study by Gallego et al., 12 (24\%) general surgeons were reported to be infected with COVID-19 [11].

The main limitation of the study is its single-center retrospective design. The results may not be generalizable to all hospitals. Patients may have been admitted to less risky hospitals. More comprehensive national and international studies are needed for the reliability of these results.

In conclusion, the number of emergency surgeries decreased by $59.1 \%$ during the pandemic. Perhaps a new algorithm is required for emergencies in these unusual situations. Based on the results of this study, to meet the increasing bed requirement during pandemic, it may be possible to follow-up patients with uncomplicated appendicitis and acute cholecystitis with antibiotics as outpatients and to perform the Taxis procedure in incarcerated hernias.

Funding The authors also declare that they have no competing financial interests.

\section{Compliance with ethical standards}

Conflict of interest The authors declare that they do not have any conflict of interest.

Ethics approval There is ethical approval from the local ethics committee (Bakirkoy Dr. Sadi Konuk Ethics Committee) and Ministry of Health approval (2020-05-20T19_56_28).

Research involving human and/or animal rights All procedures performed in this study involving human participants were in accordance with the 1964 Helsinki declaration and its latest amendments or comparable ethical standards.

Informed consent Informed consent was obtained from all individual participants of the study.

\section{References}

1. Coronavirus Disease (COVID-19) pandemic. https://www.who. int/emergencies/diseases/novel-coronavirus-2019. Accessed Mar 2020.

2. Wu F, Zhao S, Yu B, et al. A new corona virus associated with human respiratory disease in China. Nature. 2020;579(7798):265-9.

3. Ren X, Chen B, Hong Y, et al. The challenges in colorectal cancer management during COVID-19 epidemic. Ann Transl Med. 2020;8(7):498-498.

4. Hoffmann C. Treatment. In: Kamps BS, Hoffmann C (eds) Covid Reference, Edition 2020-2. SteinHauser Verlag; Amedeo, Germany; 2020. Website www.covidreference.com. Accessed 12 Apr 2020.

5. Cao W, Fang Z, Hou G, et al. The psychological impact of the COVID-19 epidemic on college students in China. Psychiatry Res. 2020;11:29-34.

6. Is it safe to go to the hospital during COVID-19 pandemic doctors say yes. https://www.heart.org/en/news/2020/05/04/. Accessed 4 May 2020.

7. Garcia S, Albaghdadi MS, Meraj PM, et al. Reduction in STsegment elevation cardiac catheterization laboratory activations in the United States during COVID-19 pandemic. J Am Coll Cardiol. 2020;75:2871-8.

8. Rodríguez-Leor O, Cid-Álvarez B, Ojeda S, et al. Impacto de la pandemia de COVID-19 sobre la actividad asistencial en cardiología intervencionista en España. REC Interv Cardiol. 2020;2:82-9.

9. Coimbra R, Edwards S, Kurihara H, et al. European Society of Trauma and Emergency Surgery (ESTES) recommendations for trauma and emergency surgery preparation during times of COVID-19 infection. Eur J Trauma Emerg Surg. 2020. https:// doi.org/10.1007/s00068-020-01364-7.

10. Wang D, Hu B, Hu C, et al. Clinical characteristics of 138 hospitalized patients with 2019 novel Coronavirus-infected pneumonia in Wuhan, China. JAMA. 2020;323(11):1061-9. https://doi. org/10.1001/jama.2020.1585. 
11. Gallego MA, Casas SG de las, Migueláñez IP et al. Impacto de la pandemia por SARS-CoV-2 sobre la actividad y profesionales de un Servicio de Cirugía General y del Aparato Digestivo en un hospital terciario. Cirugía Española. 2020; 98: 320-327. https:// doi.org/https://doi.org/10.1016/j.ciresp.2020.04.001

12. Di Saverio S, Podda M, De Simone B, et al. Diagnosis and treatment of acute appendicitis: 2020 update of the WSES Jerusalem guidelines. World J Emerg Surg. 2020;15:27. https://doi. org/10.1186/s13017-020-00306-3.

13. Ansaloni L, Pisano M, Coccolini F, et al. WSES guidelines on acute calculous cholecystitis. World J Emerg Surg. 2016;11:25. https://doi.org/10.1186/s13017-016-0082-5.

14. Tankel J, Keinan A, Blich O, et al. The decreasing incidence of acute appendicitis during COVID-19: a retrospective multi-centre study. World J Surg. 2020;44(8):2458-63. https://doi.org/10.1007/ s00268-020-05599-8.

15. Kluger Y, Ben-Ishay O, Sartelli M, et al. World society of emergency surgery study group initiative on Timing of Acute Care Surgery classification (TACS). World J Emerg Surg. 2013;8(1):17.

16. Madanelo M, Ferreira C, Nunes-Carneiro D, et al. The impact of the COVID-19 pandemic on the utilization of emergency urological services. BJU Int. 2020. https://doi.org/10.1111/bju.15109.

17. Cano-Valderrama O, Morales X, Ferrigni CJ, et al. Acute care surgery during the COVID-19 pandemic in Spain: changes in volume, causes and complications. A multicentre retrospective cohort study. Int J Surg. 2020;80:157-61. https://doi.org/10.1016/j. ijsu.2020.07.002.
18. Ten Broek RPG, Krielen P, Di Saverio S, et al. Bologna guidelines for diagnosis and management of adhesive small bowel obstruction (ASBO): 2017 update of the evidence-based guidelines from the world society of emergency surgery ASBO working group. World J Emerg Surg. 2018;13(1):24. https://doi.org/10.1186/ s13017-018-0185-2.

19. Patriti A, Baiocchi GL, Catena F, et al. Emergency general surgery in Italy during the COVID-19 outbreak: first survey from the real life. World J Emerg Surg. 2020;15:36. https://doi.org/10.1186/ s13017-020-00314-3.

20. De Simone B, Chouillard E, Di Saverio S, et al. Emergency surgery during the COVID-19 pandemic: what you need to know for practice. Ann R Coll Surg Engl. 2020;102(5):323-32.

21. East B, Pawlak M, de Beaux AC. A manual reduction of hernia under analgesia/sedation (Taxis) in the acute inguinal hernia: a useful technique in COVID-19 times to reduce the need for emergency surgery-a literature review. Hernia. 2020. https://doi. org/10.1007/s10029-020-02227-1.

22. Fang Y, Zhang H, Xie J, et al. Sensitivity of chest CT for COVID-19: comparison to RT-PCR. Radiology. 2020. https:// doi.org/10.1148/radiol.2020200432.

23. Ai T, Yang Z, Hou H, et al. Correlation of chest CT and RTPCR testing in Coronavirus disease 2019 (COVID-19) in China: a report of 1014 cases. Radiology. 2020;26:200642. https://doi. org/10.1148/radiol.202020064. 